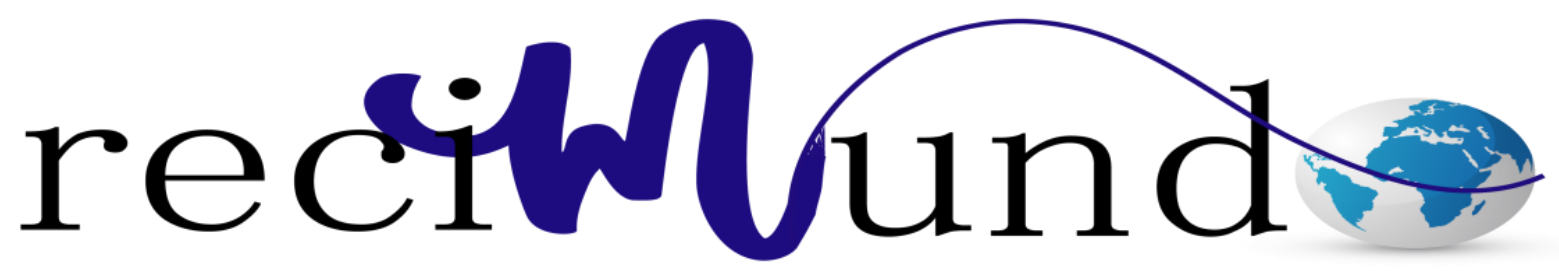

Revista Científica Mundo de la Investigación y el Conocimiento

Andrea De Los Ángeles Bravo Vidal ${ }^{\mathrm{a}}$; Tatiana Estefani Andrade Sánchez ${ }^{\mathrm{b}}$; Evelyn Lilibeth Cedeño Arteaga ${ }^{c}$; María Fernanda Castillo Silva ${ }^{\text {d }}$

Cardiopatía Isquémica, Enfermedad Prevenible

Ischemic Heart Disease, Preventable Disease

Revista Científica Mundo de la Investigación y el Conocimiento. Vol. 2 núm.3, julio, ISSN: 2588-073X, 2018, pp. 550-563

DOI: 10.26820/recimundo/2.(3).julio.2018.550-563

Editorial Saberes del Conocimiento

Recibido: 05/04/2018

Aceptado: 03/06/2018

Publicado: 30/07/2018

Correspondencia: $\underline{\text { msp.cmuso@gmail.com }}$

a. Médico; andriulinda@ gmail.com
b. Médico; tatye1989@ hotmail.com
c. Médico; $\underline{\text { evelis08@ 0tmail.com }}$
d. Médico; mafer_018@ @otmail.com 


\section{Cardiopatía Isquémica, Enfermedad Prevenible}

Vol. 2, núm. 3., (2018)

Carol Yacqueline Muso Pilchisaca; Ingrid Dora Moreno Alvarado; Néstor Neil Sánchez Haz;

Cristhian Javier Córdova Molina

\section{RESUMEN}

La Cardiopatía Isquémica produce más muertes en el mundo actualmente, incluso las proyecciones la estiman supere los índices de mortalidad para el futuro. Dada su estrecha relación con el estilo de vida de las personas, éste es el principal factor que se debe atacar en su tratamiento y prevención. La presente investigación plasma aspectos relacionados con la Cardiopatía Isquémica, con énfasis en su prevención, por cuanto a pesar de ser una enfermedad altamente prevenible los índices son sumamente elevados a nivel mundial, para ello se llevó a cabo una recopilación y revisión de material documental bibliográfico. Los factores de riesgos modificables de la enfermedad están constituidos por la hipertensión arterial, el tabaquismo, el sobrepeso y la obesidad, la inactividad física, el estrés, la diabetes, entre los principales. Se concluye que la Cardiopatía Isquémica es una enfermedad altamente prevenible, y que esta prevención radica en evitar los riesgos, se recomienda mantener una dieta sana, realizar actividad física, una vida tranquila y evitar alcohol y tabaco. Asimismo, merecen especial atención los factores de riesgos no modificables, es importante mantenerse alerta en cuanto a la edad, sexo y herencia, realizandose controles de tensión arterial, examenes para descartar azúcar en sangre, entre otros.

Palabras Claves: Trastornos, Espectro, Autista, Tratamiento, Equinoterapia. 


\title{
Cardiopatía Isquémica, Enfermedad Prevenible
}

Vol. 2, núm. 3., (2018)

Carol Yacqueline Muso Pilchisaca; Ingrid Dora Moreno Alvarado; Néstor Neil Sánchez Haz; Cristhian Javier Córdova Molina

\begin{abstract}
Ischemic heart disease produces more deaths in the world today, even projections estimate it exceeds mortality rates for the future. Given its close relationship with the lifestyle of people, this is the main factor that must be addressed in its treatment and prevention. The present investigation shows aspects related to Ischemic Heart Disease, with emphasis on its prevention, because in spite of being a highly preventable disease, the rates are extremely high worldwide, for this a compilation and review of bibliographic documentary material was carried out. . The modifiable risk factors of the disease are constituted by arterial hypertension, smoking, overweight and obesity, physical inactivity, stress, diabetes, among the main ones. It is concluded that ischemic heart disease is a highly preventable disease, and that this prevention lies in avoiding risks, it is recommended to maintain a healthy diet, perform physical activity, a quiet life and avoid alcohol and tobacco. Likewise, the non-modifiable risk factors deserve special attention, it is important to be alert in terms of age, sex and inheritance, blood pressure checks being carried out, tests to rule out blood sugar, among others.
\end{abstract}

Key Words: Disorders, Spectrum, Autistic, Treatment, Equinotherapy. 


\section{Cardiopatía Isquémica, Enfermedad Prevenible}

Vol. 2, núm. 3., (2018)

Carol Yacqueline Muso Pilchisaca; Ingrid Dora Moreno Alvarado; Néstor Neil Sánchez Haz;

Cristhian Javier Córdova Molina

\section{Introducción.}

La Organización Mundial de la Salud (2018) refiere que "Las principales causas de mortalidad en el mundo son la cardiopatía isquémica y el accidente cerebrovascular, que ocasionaron 15,2 millones de defunciones en 2016 y han sido las principales causas de mortalidad durante los últimos 15 años".

Las cifras actuales son alarmantes, pero más alarmante aún son los pronósticos, "se prevee que para el 2030, casi 23,6 millones de personas morirán por alguna enfermedad cardiovascular, principalmente por cardiopatías y accidentes cerebrovasculares”. (Organización Mundial de la Salud, s.f.)

En virtud de lo cual, esta enfermedad tan común en el mundo, irónicamente prevenible, merece especial atención, es importante su estudio, conocimiento y especial difusión de los factores de riesgo, a fin de que se trabaje en su prevención y se reduzca esta causa de muerte en el mundo.

La presente investigación plasma los aspectos generales relacionados con la Cardiopatía Isquémica con énfasis en la prevención como principal medida de reducción de las cifras de mortalidad actual y la ruptura de las proyecciones estimadas en un aumento de la enfermedad para el futuro.

\section{Materiales y métodos.}

El presente estudio se realizó mediante una recopilación y revisión de material documental bibliográfico que se utilizó para documentar y plasmar las generalidades de la 


\section{Cardiopatía Isquémica, Enfermedad Prevenible}

Vol. 2, núm. 3., (2018)

Carol Yacqueline Muso Pilchisaca; Ingrid Dora Moreno Alvarado; Néstor Neil Sánchez Haz; Cristhian Javier Córdova Molina

Cardiopatía Isquémica, con énfasis en la prevención como medida de reducción de los índices actuales de mortalidad, ya que representa una de las principales causas de muerte en el mundo, a pesar de ser altamente prevenible.

Respecto a la metodología aplicada, Ríos (2017) afirma que la investigación documental “...recurre a la consulta de documentos para obtener sus resultados”. (p. 81).

\section{Resultados.}

\section{Cardiopatía Isquémica (Generalidades)}

"Es la enfermedad ocasionada por la arteriosclerosis de las arterias coronarias, es decir, las encargadas de proporcionar sangre al músculo cardiaco (miocardio). La arteriosclerosis coronaria es un proceso lento de formación de colágeno y acumulación de lípidos (grasas) y células inflamatorias (linfocitos). Estos tres procesos provocan el estrechamiento (estenosis) de las arterias coronarias”. (Fácila Rubio, 2015)

De lo anterior se concluye que, la Cardiopatía Isquémica en palabras sencillas es un bloqueo de las arterias coronarias que impide el flujo normal, o en el peor de los casos, el paso de sangre por esta vía. Una de las principales consecuencias o complicación de esta obstrucción está representada por el infarto. 


\section{Cardiopatía Isquémica, Enfermedad Prevenible}

Vol. 2, núm. 3., (2018)

Carol Yacqueline Muso Pilchisaca; Ingrid Dora Moreno Alvarado; Néstor Neil Sánchez Haz; Cristhian Javier Córdova Molina

"Si una arteria se bloquea totalmente la falta de sangre y oxígeno puede conducir a un ataque al corazón, que destruirá una parte del miocardio. Cuando esto sucede, los impulsos eléctricos que coordinan los latidos del corazón pueden no funcionar correctamente, provocando que el corazón lata de forma irregular, más rápido o más lento. En algunos casos, las arritmias pueden ser potencialmente mortales”. (Mazzanti Mignaqui, 2018)

Esta es una de las principales causas de muerte a nivel mundial y dada su relación con los factores de riesgo y la aparición tardía de los síntomas de la enfermedad se prevee continúe aumentando sus índices.

La cardiopatía isquémica se divide en dos tipos: infarto de miocardio y angina de pecho. El primero tiene lugar cuando un trombo o coágulo en una de las arterias que suministra sangre al corazón se obstruye, impidiendo el riego sanguíneo del mismo. La gravedad radica en la cantidad de músculo cardíaco que muera, lo que se conoce como necrosis. El segundo, puede darse en dos estadios diferentes: la angina de pecho estable, que es aquella que aparece con un dolor en el tórax, pudiendo irradiarse a otras partes del cuerpo, como la mandíbula, hombro, garganta, el brazo y la muñeca izquierda. Aparece tras realizar ejercicio físico y su duración es de 1 a 15 minutos, tras el cual desaparece. Por último, la angina de pecho inestable, que es un síntoma de que existe un riesgo alto de sufrir un infarto. Al contrario que el infarto, en este caso la obstrucción de la arteria no 


\section{Cardiopatía Isquémica, Enfermedad Prevenible}

Vol. 2, núm. 3., (2018)

Carol Yacqueline Muso Pilchisaca; Ingrid Dora Moreno Alvarado; Néstor Neil Sánchez Haz; Cristhian Javier Córdova Molina

es completa, por lo que el tejido del corazón no muere porque al no producirse necrosis. (Roca, 2015)

Existen factores de riesgo que no se pueden controlar, según la Biblioteca Nacional de Medicina de los EE.UU. (2016) estos factores son:

La edad., considerando que el riesgo de cardiopatía aumenta con la edad. El género, ya que los hombres tienen un riesgo más alto de padecer cardiopatía que las mujeres que todavía están menstruando, sin embargo, después de la menopausia, el riesgo en las mujeres se acerca al de los hombres. Los genes o raza, ya que, si los padres padecieron cardiopatía, la persona tiene un riesgo de padecerlo.

Por otra parte, existen factores de riesgo que se pueden controlar, tales como la hipertensión arterial, el tabaquismo, la inactividad física, la obesidad y el sobrepeso, la diabetes, el estrés, entre los principales, sobre los cuáles es preciso prestar especial atención por cuanto sobre estos recae el mayor peso que ocasiona la enfermedad a pesar de ser prevenibles.

González y Alcalá (2010) manifiestan acerca de los factores de riesgo de la Cardiopatía Isquémica que "La hipertensión arterial es uno de los factores de riesgo más importantes para la enfermedad vascular cerebral (en especial hemorrágica), y lo es también de manera muy importante para la EIC. Como decíamos, junto con la obesidad y el tabaquismo, es una de las grandes pandemias del siglo XX. La hipertensión arterial ha 


\section{Cardiopatía Isquémica, Enfermedad Prevenible}

Vol. 2, núm. 3., (2018)

Carol Yacqueline Muso Pilchisaca; Ingrid Dora Moreno Alvarado; Néstor Neil Sánchez Haz; Cristhian Javier Córdova Molina aumentado producto del aumento en el consumo de sal, la obesidad, el sedentarismo y las dietas bajas en frutas y verduras". (p. 37)

En tratamiento de esta enfermedad implica tomar medicamentos, adoptar un estilo de vida saludable y, en el peor de los casos, someterse a cirugías. En líneas generales, los chequeos regulares forman parte importante del tratamiento.

En caso de ser fumador, el paciente debe dejar de fumar, asimismo, debe evitar ser fumador pasivo. En caso de diabetes, presión arterial alta y el colesterol alto, deben ser tratados, ya que constituyen factores de riesgo para la enfermedad. Se debe evitar alimentos procesados, llevar una dieta baja en grasas saturadas, baja en sal y baja en azúcar, consumir frutas y verduras. El ejercicio es importante para mejorar el flujo de sangre al corazón. Mantener un peso saludable y disminuir el estrés. Todos ellos son forman parte no sólo del tratamiento para la mejora sino para la prevención. Por otra parte, de ser necesario se pueden necesitar medicamentos para ayudar al corazón a trabajar más eficazmente. Por último, un tratamiento más agresivo es necesario para mejorar el flujo sanguíneo. Los procedimientos quirúrgicos comunes que pueden ayudar, tales como la angioplastia con balón y colocación de stent y la cirugía de bypass de la arteria coronaria. (Isquemia.org, 2017)

Prevención, la principal medida para la disminución de los índices de mortalidad 


\section{Cardiopatía Isquémica, Enfermedad Prevenible}

Vol. 2, núm. 3., (2018)

Carol Yacqueline Muso Pilchisaca; Ingrid Dora Moreno Alvarado; Néstor Neil Sánchez Haz; Cristhian Javier Córdova Molina

Ortega, Armas, Dueñas \& De la Noda (2015) citan a la Organización Mundial de la Salud (2002) al referirse a que “con cambios adecuados del estilo de vida se podría prevenir más de tres cuartas partes de la mortalidad cardiovascular". Asimismo, refieren que "la prevención de la Cardiopatía Isquémica aún sigue siendo un reto importante para la población, los responsables políticos y los profesionales de la salud, se define como una serie de acciones coordinadas, poblacionales e individuales, dirigidas a erradicar, eliminar o minimizar el impacto de las enfermedades cardiovasculares y la discapacidad asociada a ellas".

En líneas generales, la prevención de esta enfermedad consiste en la reducción al máximo de los factores de riesgo, estos factores son el caldo de cultivo perfecto para que aparezca la enfermedad, evitándolos se garantiza no sólo su aparecimiento sino el aparecimiento de cualquier enfermedad cardiovascular y una vida más saludable. Algunos aspectos a considerar para la prevención de la Cardiopatía Isquémica, también usados para su tratamiento, son los siguientes:

\section{Consuma una dieta sana}

La dieta debe ser saludable. Se debe evitar el sobrepeso y la obesidad para ello tomar en cuenta la ingesta calórica. "Se promoverá el consumo de fruta, verduras, legumbres, frutos secos, cereales y panes integrales y pescado (especialmente azul). La grasa saturada se sustituirá por los alimentos mencionados antes y por grasas monoinsaturadas y poliinsaturadas de origen vegetal, al objeto de reducir la ingesta calórica de la grasa 


\section{Cardiopatía Isquémica, Enfermedad Prevenible}

Vol. 2, núm. 3., (2018)

Carol Yacqueline Muso Pilchisaca; Ingrid Dora Moreno Alvarado; Néstor Neil Sánchez Haz; Cristhian Javier Córdova Molina

total a $<35 \%$ de la energía, grasas saturadas a $<7 \%$ de la energía, grasas trans a $<1 \%$ de la energía y colesterol presente en la dieta a $<300 \mathrm{mg} /$ día. La ingesta de sal debe reducirse a menos de 5 g/día, evitando el consumo de sal de mesa, limitando la cantidad de sal en la cocina y utilizando alimentos sin sal frescos y congelados; muchos alimentos procesados, incluido el pan, tienen un alto contenido de sal. Debe limitarse la ingesta de bebidas (especialmente refrescos) y alimentos con azúcar añadido, especialmente los pacientes con hipertrigliceridemia”. (Ortega, Armas , Dueñas , \& De la Noda, 2015)

\section{Haga ejercicio regularmente}

"Es un factor protector, pues aumenta el HDL-colesterol, disminuye la obesidad y la enfermedad hipertensiva. También, reduce la tensión emocional y aumenta la capacidad cardiovascular y músculo-esquelética. El mejor ejercicio para combatir la obesidad y aumentar la eficacia del corazón es el ejercicio aeróbico, es decir, de baja intensidad y de larga duración y en el que intervengan grandes grupos musculares. Se recomienda un ejercicio aeróbico de, al menos, 20-30 minutos y 3-4 días a la semana para que tenga un efecto beneficioso para el sistema cardiovascular”. (Hermosín Alcalde, Pereira, \& Núñez, 2017) 


\section{Cardiopatía Isquémica, Enfermedad Prevenible}

Vol. 2, núm. 3., (2018)

Carol Yacqueline Muso Pilchisaca; Ingrid Dora Moreno Alvarado; Néstor Neil Sánchez Haz; Cristhian Javier Córdova Molina

Evite el consumo de tabaco

Achiong Alemañy, Achiong Alemañy y Achiong Estupiñán (2015) se refieren al tabaquismo, citando a Konfino (2014), quien asegura que este “es la causa más prevenible de muerte, y uno de los factores de riesgo de cardiopatías que más inciden en la aparición de enfermedades, por lo que su control es una estrategia efectiva para reducir las muertes por la enfermedad...”.

Sin dudas el tabaco daña gravemente la salud, independientemente de cómo se consuma (cigarrillos, cigarros, pipa o tabaco para mascar). La exposición pasiva al humo del tabaco también es peligrosa. Lo bueno es que el riesgo de infarto de miocardio y las enfermedades cardiovasculares empiezan a disminuir inmediatamente después de dejar de consumir productos del tabaco y se puede reducir a la mitad en tan solo un año. (Organización Mundial de la Salud, 2015)

Verifique y controle su riesgo cardiovascular

“Un importante aspecto de la prevención de los infartos de miocardio y los accidentes cerebrovasculares es el tratamiento y asesoramiento de los pacientes con alto riesgo (aquellos con un riesgo cardiovascular a los 10 años igual o superior al 30\%) y la reducción de este. Los profesionales sanitarios pueden estimar su riesgo cardiovascular 


\section{Cardiopatía Isquémica, Enfermedad Prevenible}

Vol. 2, núm. 3., (2018)

Carol Yacqueline Muso Pilchisaca; Ingrid Dora Moreno Alvarado; Néstor Neil Sánchez Haz; Cristhian Javier Córdova Molina

con gráficas simples y darle los consejos apropiados para reducir sus factores de riesgo". (Organización Mundial de la Salud, 2015)

En este sentido es importante medir la tensión arterial, el azúcar y lípidos en sangre, el exceso de azúcar en sangre es un factor de riesgo para los infartos, además a través de estos valores se puede monitorear una aparición de diabetes.

\section{Conclusiones.}

La Cardiopatía Isquémica representa una de las principales causas de muerte a nivel mundial, irónicamente esta enfermedad es altamente prevenible.

Existen factores de riesgo que propician el aparecimiento de la enfermedad tales como la hipertensión, el sobrepeso y obesidad, la inactividad física, el estrés, la diabetes, entre otros.

Sobre estos factores es que se trabaja la prevención, es decir, evitándolos se garantiza en gran medida el aparecimiento de la enfermedad. La Organización Mundial de la Salud trabaja conjuntamente con sus países miembros en la disminución de las tasas de mortalidad por esta causa. Es importante, unificar criterios y centrarse en la prevención por cuanto constituye la mayor garantía de la reducción de los índices de muerte actuales. Desde políticas de gobierno que presten el apoyo a la difusión de información y programas de prevención, hasta las acciones personales a través de instancias públicas y privadas que las personas puedan llevar a cabo para garantizar su salud, son necesarias. 


\section{Cardiopatía Isquémica, Enfermedad Prevenible}

Vol. 2, núm. 3., (2018)

Carol Yacqueline Muso Pilchisaca; Ingrid Dora Moreno Alvarado; Néstor Neil Sánchez Haz; Cristhian Javier Córdova Molina

Incluso, es fundamental el monitoreo (chequeos regulares, exámenes de laboratorios, entre otros) ante la aparición de factores de riesgos no modificables como la edad, el sexo y la herencia, ya que de ello depende minimizar las consecuencias y controlar la enfermedad.

\section{Bibliografía.}

Achiong Alemañy, F., Achiong Alemañy, M., \& Achiong Estupiñán, F. (2015). Prevención de la cardiopatía isquémica, un desafío de la atención primaria de salud. SCIELO, 37(2). $\begin{array}{lllllll}\text { Recuperado el } 04 \quad \text { de } & \text { Agosto de }\end{array}$ http://scielo.sld.cu/scielo.php?script=sci_arttext\&pid=S1684-18242015000200005

Biblioteca Nacional de Medicina de los EE. UU. (08 de Febrero de 2016). MedlinePlus. $\begin{array}{llllll}\text { Recuperado el } 04 \text { de } & \text { Agosto de }\end{array}$ https://medlineplus.gov/spanish/ency/patientinstructions/000106.htm

Fácila Rubio, L. (Febrero de 2015). Fundación Española del Corazón. Recuperado el 03 de Agosto de 2018, de http://www.fundaciondelcorazon.com/informacion-parapacientes/enfermedades-cardiovasculares/cardiopatia-isquemica.html

González, R., \& Alcalá, J. (2010). Enfermedad isquémica del corazón, epidemiología y prevención. Medigraphic, 53(5). Recuperado el 04 de Agosto de 2018, de http://www.medigraphic.com/pdfs/facmed/un-2010/un105h.pdf

Hermosín Alcalde, A., Pereira, E., \& Núñez, A. (02 de Septiembre de 2017). Factores de riesgo de la cardiopatía isquémica. PortalesMédicos.com. Recuperado el 04 de Agosto de 2018, de https://www.revista-portalesmedicos.com/revista-medica/factores-de-riesgocardiopatia-isquemica/

Isquemia.org. (2017). Isquemia.org. Recuperado el 03 de Agosto de 2018, de http://isquemia.org/cardiopatia-isquemica/

Mazzanti Mignaqui, G. (04 de Agosto de 2018). Recuperado el 04 de Agosto de 2018, de https://www.topdoctors.es/articulos-medicos/cardiopatia-isquemica-sintomas-causasdiagnostico-y-tratamiento

Organización Mundial de la Salud. (Septiembre de 2015). Organización Mundial de la Salud. Recuperado el 03 de Agosto de 2018, de http://www.who.int/features/qa/27/es/

Organización Mundial de la Salud. (24 de Mayo de 2018). Organización Mundial de la Salud. Recuperado el 03 de Agosto de 2018, de http://www.who.int/es/news-room/factsheets/detail/the-top-10-causes-of-death 


\section{Cardiopatía Isquémica, Enfermedad Prevenible}

Vol. 2, núm. 3., (2018)

Carol Yacqueline Muso Pilchisaca; Ingrid Dora Moreno Alvarado; Néstor Neil Sánchez Haz; Cristhian Javier Córdova Molina

Organización Mundial de la Salud. (s.f.). Organización Mundial de la Salud. Recuperado el 03 de Agosto de 2018, de http://www.who.int/cardiovascular_diseases/about_cvd/es/

Ortega, Y. Y., Armas , N., Dueñas , A., \& De la Noda, R. (2015). PREVENCIÓN PRIMARIA DE LA CARDIOPATÍA ISQUÉMICA. ASPECTOS DE INTERÉS. REVISTA CUBANA DE CARDIOLOGÍA Y CIRUGÍA CARDIOVASCULAR, 21(1). Recuperado el 04 de Agosto de 2018 , http://www.revcardiologia.sld.cu/index.php/revcardiologia/article/view/566/723

Ríos, R. (09 de 2017). www.eumed.net. (E. Grupo de investigación (SEJ 309) eumed.net de la Universidad de Málaga, Ed.) Obtenido de www.eumed.net: http://www.eumed.net/libros/libro.php?id=1662

Roca, P. (15 de Enero de 2015). ONsalus. Recuperado el 04 de Agosto de 2018, de https://www.onsalus.com/cardiopatia-isquemica-causas-sintomas-y-tratamiento17496.html 